\title{
Bridge to transplantation from mechanical circulatory support: a narrative review
}

\author{
Alice L. Zhou ${ }^{1}$, Eric W. Etchill ${ }^{2}$, Katherine A. Giuliano ${ }^{2}$, Benjamin L. Shou ${ }^{1}$, Kavita Sharma ${ }^{3}$, \\ Chun W. Choi ${ }^{2}$, Ahmet Kilic ${ }^{2}$ \\ ${ }^{1}$ Johns Hopkins School of Medicine, Baltimore, MD, USA; ${ }^{2}$ Division of Cardiac Surgery, Department of Surgery, Johns Hopkins Hospital, \\ Baltimore, MD, USA; ${ }^{3}$ Division of Cardiology, Johns Hopkins School of Medicine, Baltimore, MD, USA \\ Contributions: (I) Conception and design: AL Zhou, EW Etchill, A Kilic; (II) Administrative support: None; (III) Provision of study materials or \\ patients: None; (IV) Collection and assembly of data: None; (V) Data analysis and interpretation: None; (VI) Manuscript writing: All authors; \\ (VII) Final approval of manuscript: All authors. \\ Correspondence to: Alice L. Zhou, MS. Division of Cardiac Surgery, Department of Surgery, Johns Hopkins Hospital, Baltimore, MD 21287 , USA. \\ Email: azhou18@jhmi.edu.
}

Objective: To highlight recent developments in the utilization of mechanical circulatory support (MCS) devices as bridge-to-transplant strategies and to discuss trends in MCS use following the changes to the United Network for Organ Sharing (UNOS) heart allocation system.

Background: MCS devices have played an increasingly important role in the treatment of heart failure patients. Over the past several years, technological advancements have led to new developments in MCS devices and expanding indications for MCS use. In October of 2018, the UNOS heart allocation policy was revised to prioritize higher-urgency patients, including those supported with temporary MCS devices. Since then, changes in trends of MCS utilization have been observed.

Methods: Articles from the PubMed database regarding the use of MCS devices as bridge-to-transplant strategies were reviewed.

Conclusions: Over the past decade, utilization of temporary MCS devices, which include the intraaortic balloon pump (IABP), percutaneous ventricular assist devices (pVADs), and extracorporeal membrane oxygenation (ECMO), has become increasingly common. Recent advancements in MCS include the development of pVADs that can fully unload the left ventricle (LV) as well as devices designed to provide right-sided support. Technological advancements in durable left ventricular assist devices (LVADs) have also led to improved outcomes both on the device and following heart transplantation. Following the 2018 UNOS heart allocation policy revision, the utilization of temporary MCS in advanced heart failure patients has further increased and the proportion of patients bridged directly from a temporary MCS device has exponentially risen. However, following the start of the COVID-19 pandemic, the trends have reversed, with a decrease in the percentage of patients bridged from a temporary MCS device. As long-term data following the allocation policy revision becomes available, future studies should investigate how trends in MCS use for patients with advanced heart failure continue to evolve.

Keywords: Mechanical circulatory support (MCS); heart failure; transplantation; ventricular assist device (VAD)

Submitted May 16, 2021. Accepted for publication Aug 25, 2021.

doi: $10.21037 /$ jtd-21-832

View this article at: https://dx.doi.org/10.21037/jtd-21-832 


\section{Introduction}

Heart transplantation continues to be the gold standard for patients with end-stage heart failure. However, due to increased prevalence of end-stage heart disease and the limited number of donor hearts available, the last decade has seen a $39.7 \%$ increase in the number of candidates actively awaiting transplantation (1). As a result, alternative definitive or temporizing treatment approaches utilizing various mechanical circulatory support (MCS) devices have emerged as a viable alternative in the surgical armamentarium to treat heart failure patients.

MCS devices are designed to provide hemodynamic support and help patients maintain adequate end-organ perfusion. Temporary MCS devices are intended to provide support for a number of indications, including cardiogenic shock refractory to medical therapy, high-risk percutaneous coronary interventions, myocardial recovery, and as a bridge to definitive therapy (durable MCS devices or heart transplantation). On the other hand, durable MCS devices are designed to provide long-term support and are commonly used in advanced heart failure patients as a bridge to transplantation (BTT), destination therapy (DT), or as a "bridge to decision".

In 2018, the United Network for Organ Sharing (UNOS) adult heart allocation policy was changed from a three-tier to a six-tier system, and patients supported with temporary MCS devices were assigned a higher priority status (2). Given this change, the field of MCS use has been rapidly evolving $(3,4)$. This review discusses recent developments in temporary and durable MCS devices as BTT strategies, trends in MCS utilization and outcomes over the past decade, and changes in practice following the 2018 allocation policy revision.

We present the following article in accordance with the Narrative Review reporting checklist (available at https:// dx.doi.org/10.21037/jtd-21-832).

\section{Methods}

The PubMed database was searched using combinations of the following terms: "mechanical circulatory support", "bridge to transplant", "intra-aortic balloon pump", "extracorporeal membrane oxygenation”, "percutaneous ventricular assist devices", "ventricular assist devices", "total artificial heart", "temporary mechanical circulatory support", and "durable mechanical circulatory support". Following the initial search, additional articles were added based on manual searches of the references of retrieved literature. Original articles and letters to editors containing original data published between 2005 and February of 2021 were included. Articles written in non-English were excluded.

\section{Temporary MCS devices}

Currently available temporary MCS devices include the intra-aortic balloon pump (IABP), percutaneous ventricular assist devices (pVADs), venoarterial extracorporeal membrane oxygenation (VA-ECMO), and surgically placed temporary ventricular assist devices (VADs). Device characteristics, indications for use, major contraindications, and major adverse events associated with these devices are summarized in Table 1.

\section{$I A B P$}

IABP was the earliest developed and currently the most commonly utilized form of temporary MCS (7). IABPs were initially inserted through the femoral artery and placed in the proximal descending aorta. IABP counterpulsation increases cardiac output by about $0.5 \mathrm{~L} / \mathrm{min}$ and allows for increased coronary perfusion, reduced afterload, and reduced myocardial oxygen consumption (8). Compared to more recent types of percutaneous MCS devices, the IABP provides lower hemodynamic support and does not significantly improve peripheral perfusion.

The IABP remains the most commonly used MCS device due to its low cost and ease of insertion. However, published outcomes of the IABP-SHOCK II trial, a randomized controlled trial of 600 patients with cardiogenic shock, found that the IABP resulted in no significant reduction in 30-day, 12-month, or 6-year mortality compared to medical therapy alone (9-11). The results of this trial, along with an increase in the use of other MCS devices, have led to a decrease in the use of IABPs for cardiogenic shock treatment and as a BTT therapy over the last decade (12-14).

Recently, there has been increased interest in IABPs inserted percutaneously through the axillary or subclavian arteries. This configuration allows patients to ambulate and undergo physical rehabilitation as they await transplantation. Reports have found that end-stage heart failure patients with upper-extremity IABPs have high rates of successful transplantation with significant increases in ambulatory distances following axillary insertion $(15,16)$, but no studies have directly compared outcomes in patients 


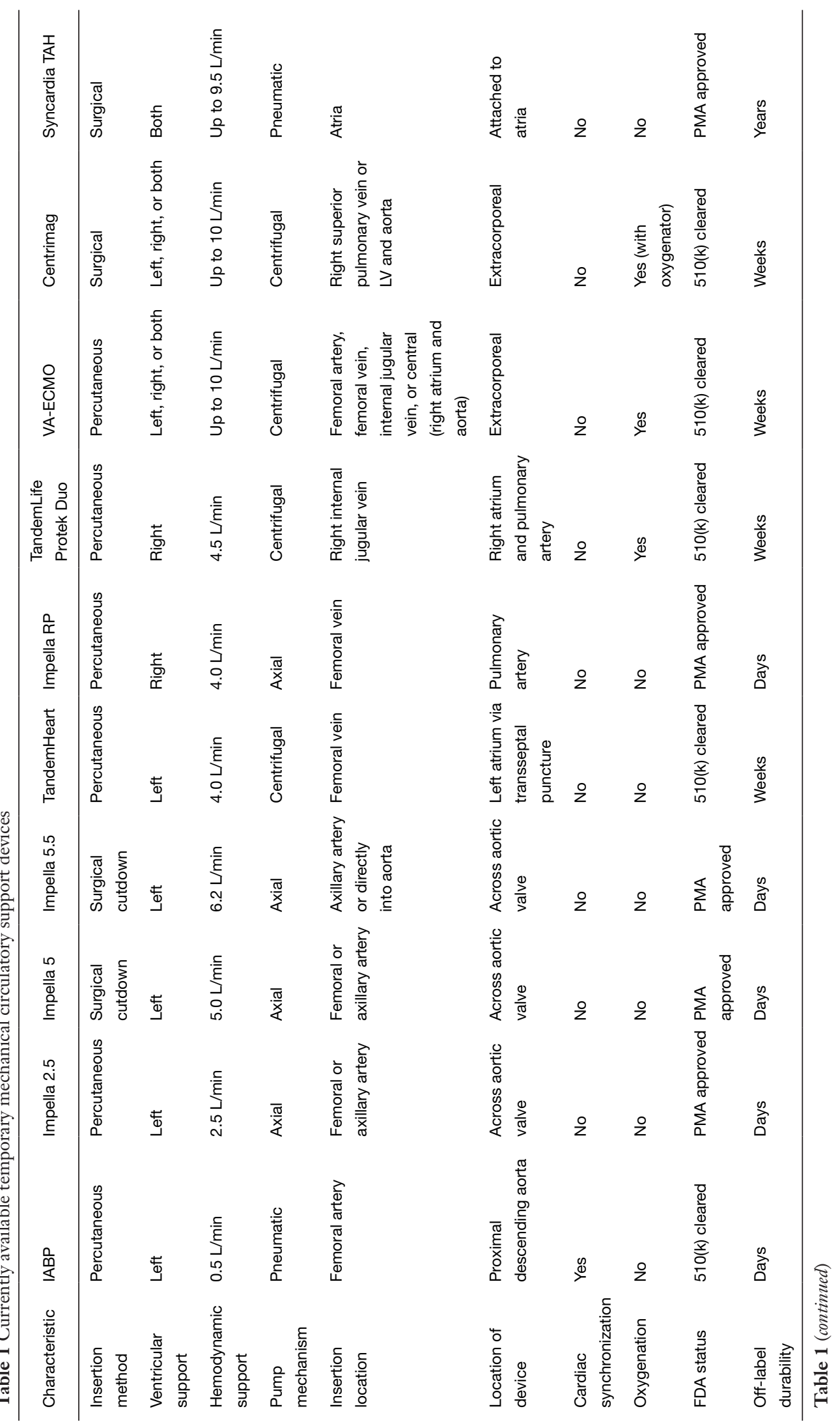




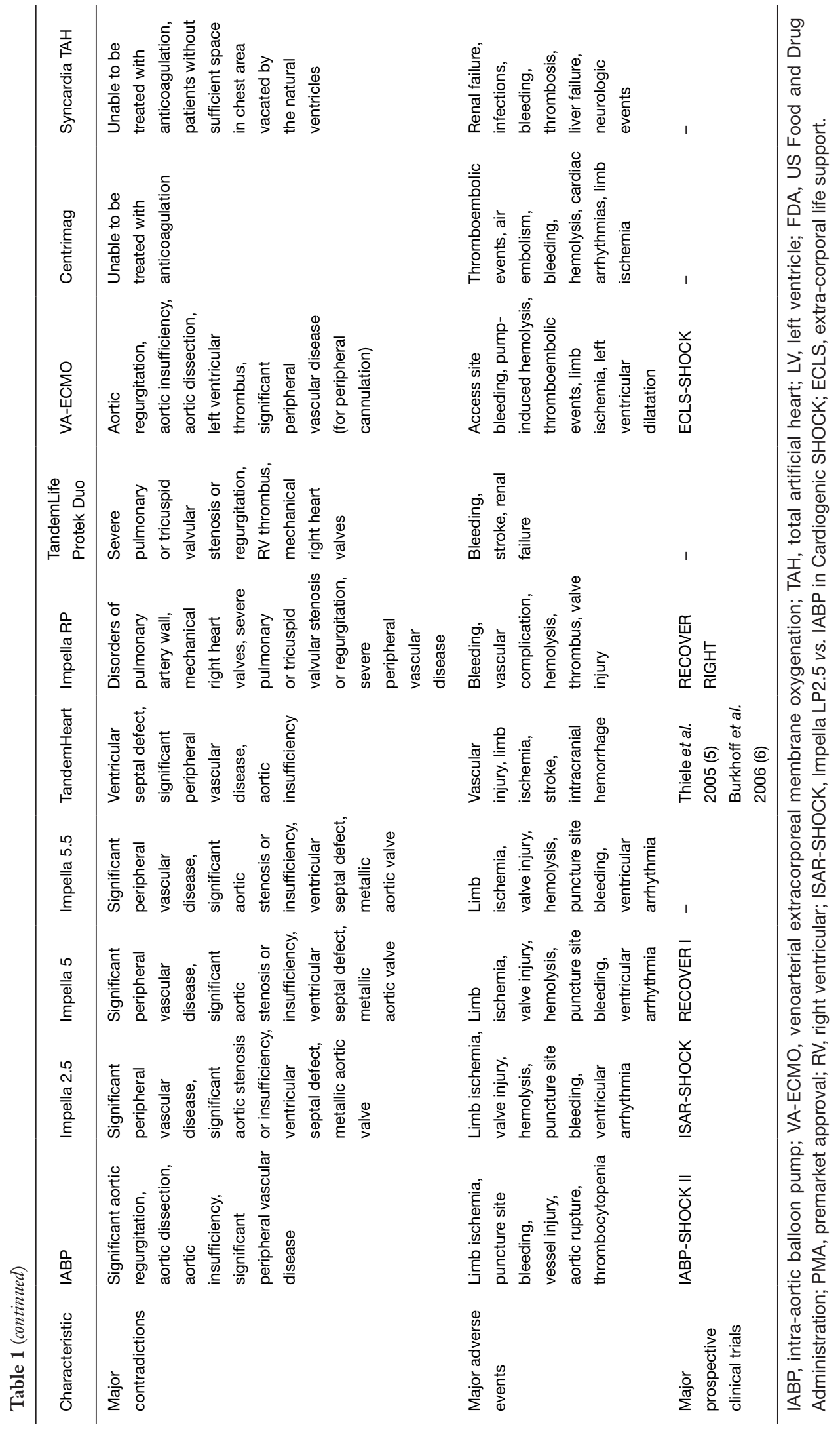


bridged with upper-extremity IABPs to those bridged with femoral IABPs.

\section{pVAD}

pVADs, such as the Impella (Abiomed, Danvers, MA, USA) and the TandemHeart (CardiacAssist, Pittsburgh, PA, USA), offer circulatory support through continuous flow pumps that can be placed either through a truly percutaneous approach or through small cut downs with short grafts. Compared to the IABP, these devices lead to greater increases in mean arterial blood pressure, more significant reductions in pulmonary capillary wedge pressure, and greater improvements in cardiac index $(6,17)$.

The Impella is a micro-axial flow device consisting of an impeller pump on a catheter. This device is placed across the aortic valve into the left ventricle (LV) and delivers non-pulsatile flow from the $L V$ to the ascending aorta. The commonly used left ventricular support devices include the Impella 2.5, Impella CP, and Impella 5.0, which provide up to $2.5,4.0$, and $5.0 \mathrm{~L} / \mathrm{min}$ of flow, respectively. The Impella 2.5 and Impella CP are typically placed percutaneously through the femoral artery, while the larger Impella 5.0 is placed through a surgical cut-down. These devices provide support by unloading the LV and decreasing myocardial oxygen consumption while improving systemic and coronary perfusion.

Recently, the Impella 5.5 with SmartAssist device was developed. This device improves upon the Impella 5.0 by providing up to $6.2 \mathrm{~L} / \mathrm{min}$ of flow, enough to fully unload the LV. In addition, the tip of the Impella 5.5 device does not have the pigtail-shaped feature of the previous Impella devices, which reduces the risk of thrombus accumulation and allows for a longer duration of implant. The SmartAssist component of the device also allows for remote monitoring and real-time calculation and display of hemodynamic parameters, such as left ventricular end-diastolic pressure, mean arterial pressure, and cardiac power output, that enable monitoring of patient status during weaning and escalation of therapy. The first experience with the Impella 5.5 was reported in 2019 when Bernhardt et al. (18) placed the device into two critically ill patients with no observed pumprelated adverse events. In 2020, Ramzy et al. (19) reported on the outcomes of the first 55 patients in the United States to receive the Impella 5.5, of which 46 (83.6\%) survived to explant and $5(9.1 \%)$ underwent heart transplantation. Further studies on the outcomes of patients with the Impella 5.5 should be performed to better understand the effectiveness of this device as a BTT therapy compared to other temporary MCS devices.

The TandemHeart is a centrifugal-flow percutaneous VAD that delivers up to $4.0 \mathrm{~L} / \mathrm{min}$ of continuous flow by removing blood from the left atrium through a transseptal puncture and transferring it to the femoral artery. The pump consists of an electromagnetic motor with a maximum speed of 7,500 rpm. Randomized controlled trials have found that the TandemHeart, compared to the IABP, provides greater hemodynamic support, but results in greater complication rates and no difference in 30-day mortality $(5,6)$. One benefit of the TandemHeart over the Impella is that the device can be repositioned to a rightatrium-to-iliofemoral-artery configuration and a membrane oxygenator can be added to provide biventricular support if the patient progresses to right ventricular (RV) failure. In practice, however, the TandemHeart is infrequently used because of the technical challenge of the transseptal puncture (20). Small case series have validated the use of TandemHeart as a BTT strategy, but more studies should be done to better understand the long-term outcomes of these patients.

Recent advancements in pVADs include the development of two devices specifically designed to provide RV support-the Impella RP (Abiomed, Danvers, MA, USA) and the Protek Duo (TandemLife, Pittsburgh, PA, USA). The Impella RP device provides RV support by pumping $>4.0 \mathrm{~L} / \mathrm{min}$ of blood from the inferior vena cava to the pulmonary artery. The prospective RECOVER RIGHT study found that the Impella RP was safe and provided RV failure patients with immediate hemodynamic support (21). Recent case studies have reported on the concurrent use of the Impella RP with left-sided assist devices as BTT strategies. Varian et al. (22) successfully used the Impella 5.0 and the Impella RP as a BTT therapy in a patient with rapidly progressive cardiac sarcoidosis. Randhawa et al. (23) reported on the first extended use of the Impella RP in a patient with a durable left ventricular assist device (LVAD) as a BTT strategy. The patient was supported by the Impella RP for 37 days and was successfully bridged to transplantation without any device-related complications.

The Protek Duo is a dual-lumen cannula that provides up to $4.5 \mathrm{~L} / \mathrm{min}$ of flow. This device is placed through the right internal jugular vein, which allows for the potential for patient ambulation. When the Protek Duo is used with an extracorporeal centrifugal-flow pump, blood is removed from the right heart and reintroduced at the pulmonary artery bifurcation. A single-center retrospective 
review of 27 patients receiving the Protek Duo after LVAD implantation found a low rate of adverse events and a 1 -year cohort survival of $81 \%$ (24), which compares well against the overall survival rate of $60 \%$ in LVAD patients requiring biventricular support (25). However, no studies have reported on the use of the Protek Duo as a bridge to transplant strategy.

\section{ECMO}

ECMO can be utilized in a veno-venous configuration for lung failure but can also provide hemodynamic support when utilized in the veno-arterial (VA) configuration. It can provide $>6 \mathrm{~L} / \mathrm{min}$ of biventricular support in addition to oxygenation and ventilation capabilities. Indications for VAECMO support include cardiogenic shock, extracorporeal cardiopulmonary resuscitation, and post-cardiotomy shock. In general, VA-ECMO is initiated via peripheral cannulation, with deoxygenated blood being removed from the femoral or internal jugular vein and oxygenated blood returned to circulation via the femoral artery. Unlike IABP and other percutaneous MCS devices, VA-ECMO increases afterload of the LV secondary to the arterial return of blood, which can increase LV end-diastolic pressure and lead to significant distention of the LV and hence myocardial ischemia. As a result, VA-ECMO may require venting of the $L V$ to avoid worsening of $\mathrm{LV}$ function in those that are in severe shock or are unlikely to recover. In recent years, a number of strategies have been developed in order to vent and hence reduce $L V$ end-diastolic pressure and distention, including concomitant use of IABP (to reduce afterload) or Impella (to reduce preload). Studies have found that treatment of cardiogenic shock with VA-ECMO and Impella to unload the LV has led to improved survival in a configuration termed "Ec-pella" (26). However, some controversy exists over whether concomitant use of IABP or Impella improves BTT outcomes in patients supported with VA-ECMO (27).

In recent years, there has been a sharp increase in the proportion of patients who are directly bridged from ECMO to heart transplantation (28). However, despite advancements in LV unloading techniques, VA-ECMO as a BTT strategy is still associated with greater rates of complications and increased early/mid-term posttransplant mortality when compared with other temporary MCS devices $(20,29,30)$. Further studies should be done to optimize LV unloading strategies and better identify patients who would benefit most from VA-ECMO as a BTT therapy.

\section{Surgically-implanted temporary VADs}

Aside from percutaneous temporary MCS devices, there are also surgically implanted devices that are placed via a median sternotomy. The most common surgically implanted temporary device is the CentriMag (Abbott Laboratories, Abbott Park, IL, USA), which is composed of a magnetically levitated pump and can provide $\mathrm{LV}, \mathrm{RV}$, or biventricular support. The CentriMag can provide up to $10 \mathrm{~L} / \mathrm{min}$ of flow and allows for patient mobility after implantation. However, implantation of the CentriMag typically requires a median sternotomy and the use of cardiopulmonary bypass, which limits its emergent use. Additionally, the CentriMag has been associated with major complications, including stroke, bleeding, and infection (31).

Recently, Takeda et al. (32) reported on a new minimally invasive technique that combines the CentriMag with ECMO and circumvents the need for a sternotomy and cardiopulmonary bypass. Compared to the conventional CentriMag biventricular assist device (BiVAD) implantation strategy, this minimally invasive technique resulted in equivalent mortality rates and decreased major bleeding events. However, no significant differences were found in rates of stroke, which remains a major complication of the CentriMag.

\section{Durable MCS devices}

\section{LVAD}

LVADs are the most widely used form of durable MCS. The first generation of LVADs were pulsatile devices, which provided patients with hemodynamic support but were limited by the need for extensive surgical dissection, frequent device exchanges, and large recipient body habitus (33). Subsequent generations of LVADs were continuousflow devices (CF-LVAD) that contained just one moving part, which allowed for a smaller pump size and greater mechanical reliability (34). CF-LVADs currently make up over $95 \%$ of all implants and have essentially replaced all other forms of durable univentricular support (35). Compared to the second generation axial-flow LVADs, the third generation devices contain centrifugal-flow pumps and are designed to be smaller and more durable. As LVADs have become more compact, there has been increasing interest in minimally invasive and sternal-sparing implantation techniques to improve patient outcomes (36-38).

Recently, the most widely used LVADs have been the HeartWare HVAD (Medtronic, Minneapolis, MN, USA) 
and the HeartMate 3 (Abbott Laboratories, Chicago, IL, USA), both of which are third-generation, centrifugal-flow LVADs. The HeartWare HVAD was FDA approved for BTT in 2012. Compared to the HeartMate II, the most widely used second-generation LVAD, the HeartWare device is smaller, lies completely within the pericardial space, and has contact-free rotation of the impeller (39). At the time of FDA approval, a randomized clinical trial investigating survival following device implantation found that the HeartWare device was noninferior to the HeartMate II device with respect to death or disabling stroke (40). The HeartMate II was found to be associated with an increased need for device replacement, explantation, and urgent transplantation, while the HeartWare device was associated with increased rates of sepsis, stroke, and heart failure (40). However, since its approval, the FDA has issued a series of Class I recalls after complaints of delayed or failure to restart after the pump stopped (41). In June of 2021, the HeartWare HVAD system was pulled from the market after evidence demonstrated higher rates of neurological adverse events and mortality relative to other LVAD systems, and patients were encouraged by Medtronic to use alternatives, such as the HeartMate 3 device $(42,43)$.

The HeartMate 3 was approved by the FDA in 2017, making it the most recent durable BTT device approved. Similar to the HeartWare, the HeartMate 3 is smaller than the previous generation of LVADs and can be fully placed within the pericardial space. The HeartMate 3 uses a magnetically levitated rotor and wide blood-flow gaps to minimize shear stress (44). In the randomized MOMENTUM 3 trial, the HeartMate 3 was found to be superior to the HeartMate II with respect to survival free of disabling stroke or device removal (45), even when looking specifically at the cohort of BTT or bridge to transplant candidacy patients (46). Compared with patients who received the HeartMate II, those who received the HeartMate 3 spent fewer days in the hospital and had lower rates of bleeding during the two years following device implantation (45). However, studies of early post-transplant outcomes in patients bridged from the HeartMate 3 found no difference in short-term survival compared with patients bridged from HeartMate $2(47,48)$.

\section{Right ventricular assist device (RVAD), BiVAD, and total artificial heart (TAH)}

Patients requiring biventricular support have poor rates of survival to transplantation (25). The current BTT therapy options for these patients include BiVADs or TAHs. BiVADs, which consist of the simultaneous use of an RVAD with an LVAD, are used in LVAD patients who develop RV failure. Currently, there are no continuous flow centrifugal pump VADs designed for right-sided use. However, there are a few pulsatile paracorporeal devices that can provide RVAD and BiVAD support, including the Thoratec Paracorporeal Ventricular Assist Device (PVAD) (Abbott Laboratories, Pleasanton, CA, USA), FDA approved in 1995 for BTT, and Abiomed AB5000 (Abiomed Inc., Danvers, MA, USA), FDA approved in 2003 (49). BiVAD strategies have also utilized off-label placement of continuous flow LVADs in a right atrial or RV position. A systematic review of right-sided use of the HeartWare found that right atrial VAD placement results in a survival advantage compared to RV VAD placement (50). Overall, BiVAD support remains infrequent and is only utilized in approximately $5 \%$ of patients with MCS devices (35).

In patients with biventricular heart failure identified preoperatively, a TAH implantation may also be used. The only TAH currently FDA approved for BTT is the SynCardia TAH (SynCardia Systems, Inc., Houston, TX, USA), a fourchambered, pulsatile device. Recent developments in the SynCardia include the Portable Freedom Driver, which allows patients to be discharged (51). The CARMAT TAH (CARMAT SA, Vélizy-Villacoublay, France) is another TAH device that is commercially available in Europe and currently being tested in clinical trials in the US. The CARMAT TAH utilizes bioprosthetic materials and sensor-based autoregulation to minimize thromboembolism and hemorrhage. Netuka et al. (52) recently reported on the successful bridge-totransplantation of 5 patients supported with CARMAT TAH after a median support time of 243 days. Overall, however, mortality rates for TAH patients remain high, with a one-year survival of less than $60 \%$ (35). TAH continues to be infrequently utilized, with just 373 implantations reported in the INTERMACS database between 2006 and 2016, compared to 17,016 continuous flow LVADs implanted during the same time period (35).

\section{Bridge-to-bridge patients}

Patients receiving temporary MCS devices who are not directly bridged to transplantation may instead be bridged to a durable MCS device. In the context of cardiogenic shock, utilization of temporary MCS devices may provide patients with hemodynamic support, which allows more time for clinical evaluation and a decision to be made, 
as well as time to correct end-organ injury secondary to cardiogenic shock. However, a study by Shah et al. (53) on post-LVAD implantation outcomes found that patients bridged from a temporary MCS device had improved hemodynamics and reversal of cardiogenic shock, but only partial end-organ recovery. Additionally, the use of a temporary MCS device was associated with a twofold increase in post-LVAD mortality, likely due to the acutely ill patient population that requires temporary MCS. A recent study by Hernandez-Montfort et al. (54) of the International Society for Heart and Lung Transplantation (ISHLT) Registry found that patients bridged to durable VADs from ECMO, in particular, resulted in lower longitudinal survival compared with patients bridged to durable VADs from other temporary MCS devices. In this study, $78 \%$ of ECMO patients had INTERMACS Profile 1 status prior to LVAD implantation, compared to just $45 \%$ in patients supported with other forms of temporary MCS. This suggests that ECMO patients are sicker prior to LVAD implantation than patients supported with other types of temporary MCS, which could explain the worse post-LVAD outcomes observed in ECMO patients.

Karamlou et al. (55) studied heart transplant outcomes in patients in the UNOS database who were double bridged to transplantation between 2000-2010. Patients who were bridged directly from an LVAD were found to have better post-transplant survival than patients who were bridged from ECMO, RVAD, or BiVAD. Meanwhile, patients who were bridged from ECMO or BiVAD to LVAD prior to transplantation experienced recovery in survival and were found to have equivalent post-transplant outcomes as those bridged from LVAD-only. No reports of a contemporary cohort of double-bridged patients have been published, and patients being double bridged from percutaneous VADs, such as the Impella or TandemHeart, have not been studied.

\section{Trends prior to the new heart allocation system}

Technological developments and advancements in the management of heart failure patients led to improvements in long term survival of patients supported with durable LVADs, particularly when comparing the 2008-2012 era with more recent years (35). Since 2013, however, LVAD survival outcomes have remained relatively unchanged. Currently, 12 -month survival is $82 \%$ and 24 -month survival is $72 \%$ (56). Post-transplant outcomes in patients bridged from an LVAD have also improved significantly. In a contemporary cohort of patients, LVAD prior to transplantation resulted in no differences in long term post-transplant outcomes when compared to patients undergoing de novo heart transplantation (57). In addition, the duration of LVAD support does not affect posttransplant outcomes (58).

As long-term outcomes of LVADs have improved, there has been increased interest in the use of LVADs as an alternative to heart transplantation for those who are ineligible for candidacy. According to the eighth annual INTERMACS report published in 2018, the number of patients receiving durable LVAD implantations increased from 4,722 in 2008-2011 to 5,400 in 2015-2016, with a significant increase in the percentage of those receiving LVADs intended for destination therapy (DT) from 28.5\% $(n=1,347)$ to $49.8 \%(n=2,687)$. Meanwhile, the percentage of LVAD patients intended for BTT decreased from $32.3 \%(n=1,525)$ in $2008-2011$ to $26.4 \%(n=1,427)$ in 2015-2016 (35). At the same time, there was an increase in the use of temporary MCS devices. The percentage of patients who received temporary MCS prior to an LVAD increased from $23.1 \%$ in the $2008-2011$ era to $28.5 \%$ in the 2012-2017 era (59).

\section{Trends following the new heart allocation system}

The UNOS heart allocation policy was revised in 2018 with the goal of better prioritizing waitlist candidates based on medical urgency, reducing clustering of candidates assigned the top-tier status, decreasing waitlist times, and providing more equitable geographic access to donors. Under the previous allocation system, patients receiving both temporary and durable VADs were equally prioritized with the highest status. However, given the increase in the number of patients supported with LVADs and the improvements in long-term LVAD outcomes, $45 \%$ of patients were listed as the highest priority status under the old allocation system (60). With the 2018 heart allocation policy change, the previous 3 -tiered system was replaced with a new 6-tiered system. The prior status $1 \mathrm{~A}$ patients were stratified into status $1-3$, and the prior status $1 \mathrm{~B}$ corresponds to the new status 4 . Under the new system, patients supported by ECMO are assigned status 1, while patients receiving IABPs and other percutaneous VADs are assigned status 2. LVAD-supported patients who have complications or are within the 30-day discretionary period are assigned status 3 , and all other LVAD-supported patients are assigned status 4 (2). 
Early studies investigating the impact of the allocation policy revision using the UNOS database have all demonstrated a decrease in time spent on the waitlist and an increase in recipient graft ischemic times due to the broader geographic distribution policy adopted $(3,4,61)$. Additionally, some studies have also found a decrease in waitlist mortality, likely attributable to the decrease in time spent on the waitlist. When looking at post-transplant outcomes following the policy revision, however, results have been inconsistent, with some studies demonstrating increased post-transplant mortality, attributed to increased transplantation of more hemodynamically unstable patients $(3,4,62)$, and other studies finding no difference in posttransplant mortality $(61,63)$. Although these studies used the UNOS database, the disparate findings may be attributed to differences in study population, time periods, and follow-up time included in the investigations. Additional studies with greater follow-up time and a larger study population should be performed to draw more definitive conclusions on the impact of the policy revision on post-transplant survival.

Under the new allocation system, patients with temporary MCS devices are assigned a higher priority status than those with durable MCS devices. Unsurprisingly, reports on transplant practices following the 2018 policy change have found an increase in patients bridged directly from temporary MCS devices and a decrease in patients bridged from an $\operatorname{LVAD}(3,64)$. A single-center analysis by Liu et al. (65) found that patients were significantly more likely to be bridged from IABP and significantly less likely to be bridged from a durable LVAD after the policy change. Additional singlecenter studies have found increases in the number of patients bridged to heart transplantation from axillary IABP (66) and Impella 5.0 (67). A national analysis using the UNOS registry conducted by Jawitz et al. (61) found similar results, with the percentage of patients bridged to transplantation with a temporary MCS device increased from $13.5 \%$ to $44.5 \%$, while the percentage of patients bridged to transplantation from a durable LVAD decreased from $41.8 \%$ to $21.2 \%$, despite an increase in LVAD implantations from 2,994 in 2018 to 3,198 in $2019(61,68)$. Other national retrospective reviews using the UNOS database found similar results, with the increase in temporary MCS BTT rates mostly due to more frequent bridging from $\mathrm{ECMO}$ and $\mathrm{a}>3 \times$ increase in bridging from $\operatorname{IABP}(60,64,65,69,70)$.

While the increase in temporary MCS BTT was not unexpected, concerns have been raised over whether this trend reflects the new prioritization of patients or whether treatment practices have changed. In the analysis by Jawitz et al. (61), rates of temporary MCS utilization in listed candidates, regardless of whether they were ultimately transplanted, were found to have doubled following the allocation policy revision, suggesting that some programs may have modified their practices to provide patients with higher priority status. A study by Parker et al. (60) retrospectively compared a pre-policy cohort and a postpolicy cohort of heart transplant candidates. Results from this study identified changes in the distribution of statuses that could be not explained by patient baseline characteristics alone. Specifically, more ECMO and IABP candidates were listed than expected, which led to more high-priority listings than anticipated. The authors of this study also found a significant difference between cardiac index measurements on the justification and registration forms, which could explain the increase in IABP listings. Additionally, Varshney et al. (71) compared practices in temporary MCS use between US transplant centers and other cardiac intensive care units (ICUs). US transplant centers in the post-revision period were found to have an increase in temporary MCS use in advanced heart failure patients complicated by cardiogenic shock. This shift in practice was not found in other cardiac ICUs, suggesting that the new UNOS allocation system may be influencing treatment practices. Based on these results, it is possible that US transplant programs have favored the utilization of IABPs over inotropes and durable VADs in order to provide patients with higher priority status. Further studies should be conducted to fully understand the effects of this shift in practice.

Interestingly, the trends seen following the 2018 allocation policy change have reversed during the COVID-19 pandemic. In the period of time immediately following the pandemic, there was a decrease in heart transplants and waitlist additions (72). At the same time, there was a decrease in the proportion of patients bridged from IABP and ECMO (73). Even eight months after the start of the pandemic when heart transplant volume and waitlist additions recovered, the percentage of patients bridged from a temporary MCS device was still lower than prior to the pandemic and decreasing steadily (74). The reversal in trends could be due to the diversion of health care resources towards patients with COVID-19 or apprehension associated with hospital visits for fear of exposure. The decrease in temporary MCS BTT may also be due to a reduction in heart failure hospitalizations 
observed during the pandemic (75).

\section{Conclusions}

The field of MCS devices as BTT therapies has been rapidly evolving. The IABP was traditionally the most commonly used form of temporary MCS. However, the development of percutaneous VADs, such as the Impella and the TandemHeart, has led to a decrease in the use of IABPs. Recent technological advancements have led to the development of percutaneous VADs that can fully unload the LV, as well as temporary MCS devices that can provide support in the setting of RV heart failure. Advancements in durable LVADs have led to improved long-term survival both on the device and after transplantation in BTT patients.

Since the 2018 UNOS heart allocation policy change, the use of temporary MCS devices as BTT strategies has increased due to both the new prioritization guidelines and changes in treatment practices. Following the policy change, the greatest increase was seen in the proportion of patients bridged using an IABP or ECMO. Interestingly, since the start of the COVID-19 pandemic, a reversal in trends was observed, and the proportion of patients bridged from an IABP or ECMO is now decreasing. Future studies should be done to determine how the trends in MCS use continue to evolve. The effects of these changes on long term outcomes should be further elucidated in order to improve practices in the management of heart failure patients.

\section{Acknowledgments}

Funding: None.

\section{Footnote}

Reporting Checklist: The authors have completed the Narrative Review reporting checklist. Available at https:// dx.doi.org/10.21037/jtd-21-832

Peer Review File: Available at https://dx.doi.org/10.21037/ jtd-21-832

Conflicts of Interest: All authors have completed the ICMJE uniform disclosure form (available at https://dx.doi. org/10.21037/jtd-21-832). The authors have no conflicts of interest to declare.
Ethical Statement: The authors are accountable for all aspects of the work in ensuring that questions related to the accuracy or integrity of any part of the work are appropriately investigated and resolved.

Open Access Statement: This is an Open Access article distributed in accordance with the Creative Commons Attribution-NonCommercial-NoDerivs 4.0 International License (CC BY-NC-ND 4.0), which permits the noncommercial replication and distribution of the article with the strict proviso that no changes or edits are made and the original work is properly cited (including links to both the formal publication through the relevant DOI and the license). See: https://creativecommons.org/licenses/by-nc-nd/4.0/.

\section{References}

1. Colvin M, Smith JM, Hadley N, et al. OPTN/SRTR 2018 Annual Data Report: Heart. Am J Transplant 2020;20 Suppl s1:340-426.

2. Adult heart allocation-OPTN. Available online: https:// optn.transplant.hrsa.gov/learn/professional-education/ adult-heart-allocation/

3. Cogswell R, John R, Estep JD, et al. An early investigation of outcomes with the new 2018 donor heart allocation system in the United States. J Heart Lung Transplant 2020;39:1-4.

4. Kilic A, Mathier MA, Hickey GW, et al. Evolving Trends in Adult Heart Transplant With the 2018 Heart Allocation Policy Change. JAMA Cardiol 2021;6:159-67.

5. Thiele H, Sick P, Boudriot E, et al. Randomized comparison of intra-aortic balloon support with a percutaneous left ventricular assist device in patients with revascularized acute myocardial infarction complicated by cardiogenic shock. Eur Heart J 2005;26:1276-83.

6. Burkhoff D, Cohen H, Brunckhorst C, et al. A randomized multicenter clinical study to evaluate the safety and efficacy of the TandemHeart percutaneous ventricular assist device versus conventional therapy with intraaortic balloon pumping for treatment of cardiogenic shock. Am Heart J 2006;152:469.e1-8.

7. Enezate T, Eniezat M, Thomas J. Utilization and Outcomes of Temporary Mechanical Circulatory Support Devices in Cardiogenic Shock. Am J Cardiol 2019;124:505-10.

8. Scheidt S, Wilner G, Mueller H, et al. Intra-aortic balloon counterpulsation in cardiogenic shock. Report of a cooperative clinical trial. N Engl J Med 1973;288:979-84. 
9. Thiele H, Zeymer U, Neumann FJ, et al. Intraaortic balloon support for myocardial infarction with cardiogenic shock. N Engl J Med 2012;367:1287-96.

10. Thiele H, Zeymer U, Neumann FJ, et al. Intra-aortic balloon counterpulsation in acute myocardial infarction complicated by cardiogenic shock (IABP-SHOCK II): final 12 month results of a randomised, open-label trial. Lancet 2013;382:1638-45.

11. Thiele H, Zeymer U, Thelemann N, et al. Intraaortic Balloon Pump in Cardiogenic Shock Complicating Acute Myocardial Infarction: Long-Term 6-Year Outcome of the Randomized IABP-SHOCK II Trial. Circulation 2019;139:395-403.

12. Shah M, Patnaik S, Patel B, et al. Trends in mechanical circulatory support use and hospital mortality among patients with acute myocardial infarction and noninfarction related cardiogenic shock in the United States. Clin Res Cardiol 2018;107:287-303.

13. Panhwar MS, Gupta T, Karim A, et al. Trends in the Use of Short-Term Mechanical Circulatory Support in the United States - An Analysis of the 2012 - 2015 National Inpatient Sample. Struct Heart 2019;3:499-506.

14. Duran A, Nguyen DT, Graviss EA, et al. Waitlist and posttransplant outcomes in patients listed with intra-aortic balloon pump for heart transplant: United Network for Organ Sharing registry. Int J Artif Organs 2020;43:606-13.

15. Nwaejike N, Son AY, Milano CA, et al. Is there a role for upper-extremity intra-aortic balloon counterpulsation as a bridge-to-recovery or a bridge-to-transplant in the treatment of end-stage heart failure?. Interact Cardiovasc Thorac Surg 2017;25:654-8.

16. Nishida H, Koda Y, Kalantari S, et al. Outcomes of Ambulatory Axillary Intraaortic Balloon Pump as a Bridge to Heart Transplantation. Ann Thorac Surg 2021;111:1264-70.

17. Seyfarth M, Sibbing D, Bauer I, et al. A randomized clinical trial to evaluate the safety and efficacy of a percutaneous left ventricular assist device versus intraaortic balloon pumping for treatment of cardiogenic shock caused by myocardial infarction. J Am Coll Cardiol 2008;52:1584-8.

18. Bernhardt AM, Hakmi S, Sinning C, et al. A newly developed transaortic axial flow ventricular assist device: Early clinical experience. J Heart Lung Transplant 2019;38:466-7.

19. Ramzy D, Soltesz E, Anderson M. New Surgical Circulatory Support System Outcomes. ASAIO J 2020;66:746-52.
20. Moonsamy P, Axtell AL, Ibrahim NE, et al. Survival After Heart Transplantation in Patients Bridged With Mechanical Circulatory Support. J Am Coll Cardiol 2020;75:2892-905.

21. Anderson MB, Goldstein J, Milano C, et al. Benefits of a novel percutaneous ventricular assist device for right heart failure: The prospective RECOVER RIGHT study of the Impella RP device. J Heart Lung Transplant 2015;34:1549-60.

22. Varian $\mathrm{K}, \mathrm{Xu} \mathrm{WD}$, Lin $\mathrm{W}$, et al. Minimally invasive biventricular mechanical circulatory support with Impella pumps as a bridge to heart transplantation: a first-in-theworld case report. ESC Heart Fail 2019;6:552-4.

23. Randhawa VK, Hoffman K, Bock A, et al. Impella RP as a bridge to cardiac transplant for refractory late right ventricular failure in setting of left ventricular assist device. ESC Heart Fail 2020;7:1972-5.

24. Salna M, Garan AR, Kirtane AJ, et al. Novel percutaneous dual-lumen cannula-based right ventricular assist device provides effective support for refractory right ventricular failure after left ventricular assist device implantation. Interact Cardiovasc Thorac Surg 2020;30:499-506.

25. Kirklin JK, Naftel DC, Pagani FD, et al. Seventh INTERMACS annual report: 15,000 patients and counting. J Heart Lung Transplant 2015;34:1495-504.

26. Pappalardo F, Schulte C, Pieri M, et al. Concomitant implantation of Impella ${ }^{\circledR}$ on top of veno-arterial extracorporeal membrane oxygenation may improve survival of patients with cardiogenic shock. Eur J Heart Fail 2017;19:404-12.

27. Barge-Caballero G, Castel-Lavilla MA, Almenar-Bonet L, et al. Venoarterial extracorporeal membrane oxygenation with or without simultaneous intra-aortic balloon pump support as a direct bridge to heart transplantation: results from a nationwide Spanish registry. Interact Cardiovasc Thorac Surg 2019;29:670-7.

28. Zalawadiya S, Fudim M, Bhat G, et al. Extracorporeal membrane oxygenation support and post-heart transplant outcomes among United States adults. J Heart Lung Transplant 2017;36:77-81.

29. Fukuhara S, Takeda K, Kurlansky PA, et al. Extracorporeal membrane oxygenation as a direct bridge to heart transplantation in adults. J Thorac Cardiovasc Surg 2018;155:1607-1618.e6.

30. Yin MY, Wever-Pinzon O, Mehra MR, et al. Posttransplant outcome in patients bridged to transplant with temporary mechanical circulatory support devices. J Heart Lung Transplant 2019;38:858-69. 
31. Cevasco MR, Li B, Han J, et al. Adverse Event Profile Associated with Prolonged Use of CentriMag Ventricular Assist Device for Refractory Cardiogenic Shock. ASAIO J 2019;65:806-11.

32. Takeda K, Garan AR, Ando M, et al. Minimally invasive CentriMag ventricular assist device support integrated with extracorporeal membrane oxygenation in cardiogenic shock patients: a comparison with conventional CentriMag biventricular support configuration. Eur J Cardiothorac Surg 2017;52:1055-61.

33. Baldwin JT, Robbins RC; National Heart, Lung, and Blood Institute Working Group. Executive summary for the National Heart, Lung, and Blood Institute Working Group on next generation ventricular assist devices for destination therapy. Semin Thorac Cardiovasc Surg 2005;17:369-71.

34. Miller LW, Pagani FD, Russell SD, et al. Use of a continuous-flow device in patients awaiting heart transplantation. N Engl J Med 2007;357:885-96.

35. Kirklin JK, Pagani FD, Kormos RL, et al. Eighth annual INTERMACS report: Special focus on framing the impact of adverse events. J Heart Lung Transplant 2017;36:1080-6.

36. Carrozzini M, Bejko J, Gerosa G, et al. Bilateral minithoracotomy approach for minimally invasive implantation of HeartMate 3. Artif Organs 2019;43:593-5.

37. Kocabeyoglu SS, Kervan U, Sert DE, et al. Is it Possible to Implant HeartMate 3 Less Invasively? New Pump, New Approach. Artif Organs 2018;42:1132-8.

38. Wood KL, Ayers BC, Sagebin F, et al. Complete SternalSparing HeartMate 3 Implantation: A Case Series of 10 Consecutive Patients. Ann Thorac Surg 2019;107:1160-5.

39. Larose JA, Tamez D, Ashenuga M, et al. Design concepts and principle of operation of the HeartWare ventricular assist system. ASAIO J 2010;56:285-9.

40. Rogers JG, Pagani FD, Tatooles AJ, et al. Intrapericardial Left Ventricular Assist Device for Advanced Heart Failure. N Engl J Med 2017;376:451-60.

41. Health C for D and R. Medtronic Recalls HVAD Pump Implant Kits Due to Delayed or Failed Restart After the Pump is Stopped. FDA 2021. Available online: https:// www.fda.gov/medical-devices/medical-device-recalls/ medtronic-recalls-hvad-pump-implant-kits-due-delayedor-failed-restart-after-pump-stopped

42. Commissioner O of the. FDA Alerts Health Care Providers to Stop New Implants of Certain Ventricular Assist Device System. FDA 2021. Available online: https:// www.fda.gov/news-events/press-announcements/fda- alerts-health-care-providers-stop-new-implants-certainventricular-assist-device-system

43. Numan L, Ramjankhan FZ, Oberski DL, et al. Propensity score-based analysis of long-term outcome of patients on HeartWare and HeartMate 3 left ventricular assist device support. ESC Heart Fail 2021;8:1596-603.

44. Bourque K, Cotter C, Dague C, et al. Design Rationale and Preclinical Evaluation of the HeartMate 3 Left Ventricular Assist System for Hemocompatibility. ASAIO J 2016;62:375-83.

45. Mehra MR, Uriel N, Naka Y, et al. A Fully Magnetically Levitated Left Ventricular Assist Device - Final Report. N Engl J Med 2019;380:1618-27.

46. Goldstein DJ, Naka Y, Horstmanshof D, et al. Association of Clinical Outcomes With Left Ventricular Assist Device Use by Bridge to Transplant or Destination Therapy Intent: The Multicenter Study of MagLev Technology in Patients Undergoing Mechanical Circulatory Support Therapy With HeartMate 3 (MOMENTUM 3) Randomized Clinical Trial. JAMA Cardiol 2020;5:411-9.

47. Suarez-Pierre A, Lui C, Zhou X, et al. Early Outcomes After Heart Transplantation in Recipients Bridged With a HeartMate 3 Device. Ann Thorac Surg 2019;108:467-73.

48. Mueller M, Hoermandinger C, Richter G, et al. Retrospective 1-year outcome follow-up in 200 patients supported with HeartMate 3 and HeartWare left ventricular assist devices in a single centre. Eur J Cardiothorac Surg 2020;57:1160-5.

49. Hsu PL, Parker J, Egger C, et al. Mechanical circulatory support for right heart failure: current technology and future outlook. Artif Organs 2012;36:332-47.

50. Maynes EJ, O'Malley TJ, Patel P, et al. Right atrial versus right ventricular HeartWare HVAD position in patients on biventricular HeartWare HVAD support: A systematic review. Artif Organs 2020;44:926-34.

51. Shah NR, Jaroszewski DE, Ashfaq A, et al. SynCardia Portable Freedom Driver: A Single-Center Experience With 11 Patients. Innovations (Phila) 2015;10:188-94.

52. Netuka I, Pya Y, Bekbossynova M, et al. Initial bridge to transplant experience with a bioprosthetic autoregulated artificial heart. J Heart Lung Transplant 2020;39:1491-3.

53. Shah P, Pagani FD, Desai SS, et al. Outcomes of Patients Receiving Temporary Circulatory Support Before Durable Ventricular Assist Device. Ann Thorac Surg 2017;103:106-12.

54. Hernandez-Montfort JA, Xie R, Ton VK, et al. Longitudinal impact of temporary mechanical circulatory support on durable ventricular assist device outcomes: 
An IMACS registry propensity matched analysis. J Heart

Lung Transplant 2020;39:145-56.

55. Karamlou T, Gelow J, Diggs BS, et al. Mechanical circulatory support pathways that maximize post-heart transplant survival. Ann Thorac Surg 2013;95:480-5; discussion 485 .

56. Teuteberg JJ, Cleveland JC Jr, Cowger J, et al. The Society of Thoracic Surgeons Intermacs 2019 Annual Report: The Changing Landscape of Devices and Indications. Ann Thorac Surg 2020;109:649-60.

57. Suarez-Pierre A, Zhou X, Fraser CD 3rd, et al. Survival and Functional Status After Bridge-to-Transplant with a Left Ventricular Assist Device. ASAIO J 2019;65:661-7.

58. Grimm JC, Magruder JT, Crawford TC, et al. Duration of Left Ventricular Assist Device Support Does Not Impact Survival After US Heart Transplantation. Ann Thorac Surg 2016;102:1206-12.

59. Kormos RL, Cowger J, Pagani FD, et al. The Society of Thoracic Surgeons Intermacs database annual report: Evolving indications, outcomes, and scientific partnerships. J Heart Lung Transplant 2019;38:114-26.

60. Parker WF, Chung K, Anderson AS, et al. Practice Changes at U.S. Transplant Centers After the New Adult Heart Allocation Policy. J Am Coll Cardiol 2020;75:2906-16.

61. Jawitz OK, Fudim M, Raman V, et al. Reassessing Recipient Mortality Under the New Heart Allocation System: An Updated UNOS Registry Analysis. JACC Heart Fail 2020;8:548-56.

62. Trivedi JR, Slaughter MS. "Unintended" Consequences of Changes in Heart Transplant Allocation Policy: Impact on Practice Patterns. ASAIO J 2020;66:125-7.

63. Goff RR, Uccellini K, Lindblad K, et al. A change of heart: Preliminary results of the US 2018 adult heart allocation revision. Am J Transplant 2020;20:2781-90.

64. Nordan T, Hironaka CE, Kawabori M. Temporary Mechanical Circulatory Support as a Bridge to Transplant: Return of the Intra-Aortic Balloon Pump. JACC Heart Fail 2020;8:785-6.

65. Liu J, Yang BQ, Itoh A, et al. Impact of New UNOS Allocation Criteria on Heart Transplant Practices and

Cite this article as: Zhou AL, Etchill EW, Giuliano KA, Shou BL, Sharma K, Choi CW, Kilic A. Bridge to transplantation from mechanical circulatory support: a narrative review. J Thorac Dis 2021;13(12):6911-6923. doi: 10.21037/jtd-21-832
Outcomes. Transplant Direct 2020;7:e642.

66. Albert CL, Hsich E, Unai S, et al. The Safety and Efficacy Intra-Aortic Balloon Pump as a Bridge to Transplant Strategy under the New UNOS Allocation. J Card Fail 2019;25:S161.

67. Kearns MJ, Chou L, Aguillon M, et al. Impella 5.0 as a Bridge to Cardiac Transplantation before and after Reclassification of the United Network for Organ Sharing Heart Allocation Criteria. J Heart Lung Transplant 2020;39:S335.

68. Molina EJ, Shah P, Kiernan MS, et al. The Society of Thoracic Surgeons Intermacs 2020 Annual Report. Ann Thorac Surg 2021;111:778-92.

69. Huckaby LV, Seese LM, Mathier MA, et al. IntraAortic Balloon Pump Bridging to Heart Transplantation: Impact of the 2018 Allocation Change. Circ Heart Fail 2020;13:e006971.

70. Hanff TC, Harhay MO, Kimmel SE, et al. Trends in Mechanical Support Use as a Bridge to Adult Heart Transplant Under New Allocation Rules. JAMA Cardiol 2020;5:728-9.

71. Varshney AS, Berg DD, Katz JN, et al. Use of Temporary Mechanical Circulatory Support for Management of Cardiogenic Shock Before and After the United Network for Organ Sharing Donor Heart Allocation System Changes. JAMA Cardiol 2020;5:703-8.

72. DeFilippis EM, Sinnenberg L, Reza N, et al. Trends in US Heart Transplant Waitlist Activity and Volume During the Coronavirus Disease 2019 (COVID-19) Pandemic. JAMA Cardiol 2020;5:1048-52.

73. Nordan T, Couper GS, Chen FY, et al. Patterns of Use of Temporary Mechanical Circulatory Support as a Bridge to Transplant During the Coronavirus Disease 2019 Pandemic. J Card Fail 2020;26:902-4.

74. Kim ST, Hadaya J, Tran Z, et al. Impact of the Coronavirus Disease 2019 Pandemic on Utilization of Mechanical Circulatory Support As Bridge to Heart Transplantation. ASAIO J 2021;67:382-4.

75. Hall ME, Vaduganathan M, Khan MS, et al. Reductions in Heart Failure Hospitalizations During the COVID-19 Pandemic. J Card Fail 2020;26:462-3. 